\title{
Barbiturates Induce Mitochondrial Depolarization and Potentiate Excitotoxic Neuronal Death
}

\author{
Christopher M. Anderson, ${ }^{1}$ Becky A. Norquist, ${ }^{1}$ Sabino Vesce, ${ }^{2}$ David G. Nicholls, ${ }^{2}$ William H. Soine, ${ }^{3}$ \\ Shumin Duan, ${ }^{1}$ and Raymond A. Swanson ${ }^{1}$ \\ ${ }^{1}$ Department of Neurology, University of California, San Francisco, and Department of Veterans Affairs Medical Center, \\ San Francisco, California 94121, ${ }^{2}$ Buck Institute for Research on Aging, Novato, California 94945, and ${ }^{3}$ Department of \\ Medicinal Chemistry, Virginia Commonwealth University, Richmond, Virginia 23298
}

Barbiturates are widely used as anesthetics, anticonvulsants, and neuroprotective agents. However, barbiturates may also inhibit mitochondrial respiration, and mitochondrial inhibitors are known to potentiate NMDA receptor-mediated neurotoxicity. Here we used rat cortical cultures to examine the effect of barbiturates on neuronal mitochondria and responses to NMDA receptor stimulation. The barbiturates tested, secobarbital, amobarbital, and thiamylal, each potentiated NMDA-induced neuron death at barbiturate concentrations relevant to clinical and experimental use (100-300 $\mu \mathrm{M})$. By using rhodamine-123 under quenching conditions, barbiturates in this concentration range were shown to depolarize neuronal mitochondria and greatly amplify NMDAinduced mitochondrial depolarization. Barbiturate-induced mitochondrial depolarization was increased by the ATP synthase inhibitor oligomycin, indicating that barbiturates act by inhibiting electron transport sufficiently to cause ATP synthase reversal. Barbiturates similarly amplified the effects of NMDA on cytoplas- mic free calcium concentrations. The cell-impermeant barbiturate $N$-glucoside amobarbital did not influence mitochondrial potential or potentiate NMDA neurotoxicity or calcium responses. However, all of the barbiturates attenuated NMDA-induced calcium elevations and cell death when present at millimolar concentrations. Whole-cell patch-clamp studies showed that these effects may be attributable to actions at the cell membrane, resulting in a block of NMDA-induced current flux at millimolar barbiturate concentrations. Together, these findings reconcile previous reports of opposing effects on barbiturates on NMDA neurotoxicity and show that barbiturate effects on neuronal mitochondria can be functionally significant. Effects of barbiturates on neuronal mitochondria should be considered in experimental and clinical application of these drugs.

Key words: amobarbital; N-glucoside amobarbital; secobarbital; calcium; glutamate; NMDA; rhodamine-123; fura-2
Barbiturates reduce glutamate-induced neuronal depolarization (Barker and Ransom, 1978), block voltage-dependent $\mathrm{Ca}^{2+}$ channels (Werz and Macdonald, 1985), attenuate the $\mathrm{Ca}^{2+}$ influx of NMDA-induced currents (Daniell, 1994; Charlesworth et al., 1995), and depress neuronal activity and metabolism (Sokoloff et al., 1977; Steen and Michenfelder, 1980). These actions suggest that barbiturates should effectively reduce excitotoxic neuronal injury, particularly under conditions of energy deprivation. Therefore, it is surprising that barbiturates do not attenuate neuronal death in cell-culture models of glucose or combined oxygen-glucose deprivation (Giffard et al., 1993) that produce NMDA receptor-dependent toxicity.

The present study examines whether the potential neuroprotective effects of barbiturates may be limited or outweighed by inhibitory effects on mitochondrial function. Barbiturates have long been known to depress mitochondrial respiration (Aldrich and Parker, 1960; Chance and Hollunger, 1963). In isolated mitochon-

\footnotetext{
Received April 4, 2002; revised Aug. 22, 2002; accepted Aug. 22, 2002.

This work was supported by American Heart Association Grant-in-Aid 9750537N, National Institutes of Health Grant RO1 NS31914, the Department of Veterans Affairs, the Canadian Institutes of Health Research, and the Heart and Stroke Foundation of Canada. We thank Dr. Conrad Alano and Elizabeth Gum for advice and technical assistance.

Correspondence should be addressed to Dr. Raymond A. Swanson, Neurology Service (127), Veterans Affairs Medical Center, 4150 Clement Street, San Francisco, CA 94121. E-mail: ray@itsa.ucsf.edu.

S. Duan's present address: Institute of Neuroscience, Chinese Academy of Sciences, Shanghai 200031, China.

Copyright (C) 2002 Society for Neuroscience $0270-6474 / 02 / 229203-07 \$ 15.00 / 0$
}

dria, compounds of the oxybarbiturate class (including secobarbital, amobarbital, and amytal) inhibit respiration by interfering with complex 1 of the electron transport chain (Aldrich and Parker, 1960). Thiobarbiturates (including thiamylal and thiopental) may additionally have an "uncoupling" effect on oxidative phosphorylation (Aldrich and Parker, 1960; Chance and Hollunger, 1963). These effects are incomplete and do not appreciably depress cell ATP levels under basal metabolic conditions; however, barbiturate effects on cellular energy metabolism may become physiologically significant during periods of reduced substrate delivery or high energy demand (Swanson and Seid, 1998).

Barbiturate actions on mitochondria could be particularly important during NMDA receptor activation. Mitochondrial inhibitors have been shown to amplify NMDA receptor-mediated intracellular calcium $\left(\left[\mathrm{Ca}^{2+}\right]_{\mathrm{i}}\right)$ elevations, by both impairment of intracellular calcium homeostasis and plasma membrane depolarization and resultant increased $\mathrm{Ca}^{2+}$ flux through NMDAgated cation channels (White and Reynolds, 1995; Greene et al., 1998). Several mitochondrial inhibitors can potentiate NMDA receptor activation and excitotoxic neuronal death (Novelli et al., 1988; Beal, 1992; Greene and Greenamyre, 1996; Greene et al., 1998), suggesting that barbiturates might have similar effects. In this study, we used cortical neuron cultures to examine the effects of barbiturates on NMDA-mediated mitochondrial depolarization, intracellular $\mathrm{Ca}^{2+}$ elevations, and cell death. The findings demonstrate that barbiturates have direct effects on neuronal mitochondria and thereby potentiate responses to NMDA. 


\section{MATERIALS AND METHODS}

Materials. $\quad 5(R / S)$-5-ethyl-1-(1- $\beta$-D-glucopyranosyl)-5-(3-methylbutyl)2,3,6- $(1 \mathrm{H}, 3 \mathrm{H}, 5 \mathrm{H})$-pyrimidinetrione $(N$-glucoside amobarbital) was synthesized as described previously (Soine et al., 1986). All other reagents were purchased from Sigma (St. Louis, MO) except where otherwise stated.

Astrocyte-neuron cocultures. Cortical cultures were prepared from newborn and neonatal rats using protocols approved by the San Francisco Veterans Affairs Medical Center animal use review committee and following National Institutes of Health guidelines. Cocultures were prepared by seeding neurons onto a pre-existing astrocyte layer. Astrocyte cultures were prepared from cortices harvested from 1-d-old Sprague Dawley rats (Simonsen, Gilroy, CA). After removal of meninges, the cells were dissociated by incubation in papain/DNase, followed by trituration. The dissociated cells were washed, suspended in Eagle's minimum essential medium (MEM) with 10\% fetal bovine serum (FBS; Hyclone, Ogden, UT) and $2 \mathrm{~mm}$ glutamine, and plated in Falcon 24 well tissue culture plates at an approximate density of $5 \times 10^{4} \mathrm{cells} / \mathrm{cm}^{2}$. For some studies, the astrocytes were plated onto glass coverslips placed on the bottoms of the culture wells. The cultures were maintained in a humidified, $5 \% \mathrm{CO}_{2}, 37^{\circ} \mathrm{C}$ incubator and received a medium exchange every 7 d. Neurons prepared from fetal (embryonic day 16) rats were plated onto the astrocytes after 14-20 d, when the astrocytes form a confluent monolayer. The fetal rat forebrain cortices were removed, and cells were dissociated by the same method used for the astrocyte preparations. These cells were plated at an approximate density of $1 \times 10^{5}$ cells $/ \mathrm{cm}^{2}$, and the resulting cocultures were maintained in a $5 \% \mathrm{CO}_{2}$ atmosphere. Proliferation of other cell types was inhibited by the addition of $10 \mu \mathrm{M}$ cytosine arabinoside $3 \mathrm{~d}$ after plating. This medium was replaced after 48 hr with glial-conditioned medium prepared by placing MEM with $2 \mathrm{mM}$ glutamine and 5\% FBS into a flask of confluent cortical astrocytes for 72 hr. The coculture medium was subsequently exchanged with fresh glialconditioned media every $7 \mathrm{~d}$ and on the evening before use of the cells. Experiments were conducted when the neurons were 18-22 d in vitro.

Experimental media. Experimental incubations were performed at $37^{\circ} \mathrm{C}$ in a balanced salt solution (BSS) containing (in mM): $135 \mathrm{NaCl}, 3.1 \mathrm{KCl}, 1.2$ $\mathrm{CaCl}_{2}, 1.2 \mathrm{MgSO}_{4}, 0.5 \mathrm{KH}_{2} \mathrm{PO}_{4}, 5$ 1,4-piperazinediethanesulfonic acid, and 2 glucose, $\mathrm{pH}$ 7.2. All drugs were added from concentrated iso-osmolarbuffered stock solutions, $\mathrm{pH}$ 7.2.

Neuron death. Cells were washed by three partial $(85 \%)$ exchanges into prewarmed BSS containing $0.1 \%$ bovine serum albumin (BSA). Barbiturates were added 5 min before additions of NMDA or $\mathrm{H}_{2} \mathrm{O}_{2}$. Incubations were terminated after 5 min with NMDA or 10 min with $\mathrm{H}_{2} \mathrm{O}_{2}$ by washing back into MEM containing $0.1 \%$ BSA. Neuronal death was determined 18-24 hr after NMDA exposures by measurement of lactate dehydrogenase (LDH) in the medium (Koh and Choi, 1987). LDH values corresponding to $100 \%$ neuronal death were established for each 24 well culture plate by treating four of the wells with $10 \mathrm{~mm}$ NMDA to kill all neurons. The LDH values from the other 20 wells were then normalized to these values to express results as a percentage of neuronal death. Subtraction of background LDH values, representing neuronal death under control conditions, was performed only where noted. Propidium iodide (PI) staining (Edidin, 1970) was used to assess neuron death 18-24 hr after $\mathrm{H}_{2} \mathrm{O}_{2}$ exposure and in selected NMDA-treated cultures to corroborate the LDH results and confirm that $\mathrm{LDH}$ release reflected only neuronal death. PI $(0.01 \mathrm{mg} / \mathrm{ml})$ was added to each well, and both live and dead (PI stained) cells were counted in random optical fields in each of four quadrants, totaling $>500$ cells per well. Neurons were distinguished from the underlying glial layer by a phase-bright, processbearing appearance under phase-contrast optics (Ying et al., 1999).

Intracellular calcium. Intracellular cytoplasmic $\mathrm{Ca}^{2+}$ measurements were performed by fura- 2 ratiometric imaging. Cultures on coverslips were loaded with $8 \mu \mathrm{M}$ fura-2 acetoxymethyl ester (Molecular Probes, Eugene, OR) for $15 \mathrm{~min}$ at room temperature, washed, and then treated with barbiturates and NMDA as indicated. Coverslips were placed in a chamber on an Olympus inverted microscope (Scientific Instruments, Sunnyvale, CA) with a $40 \times$ objective for fluorescence imaging. Data were recorded from 5 to 20 cells per coverslip and were averaged from three or more coverslips. Cells were excited alternately at 340 and 380 $\mathrm{nm}$, and emission was recorded at $510 \mathrm{~nm}$. Calcium measurements were determined as the ratio of emission intensities at 340 and $380 \mathrm{~nm}$ excitation wavelengths by using standard imaging techniques and analysis with Metafluor software from Universal Imaging (West Chester, PA). The NMDA-induced change in fluorescence ratio was integrated over time for each imaged neuron using an in-house macro program for Microsoft Excel (Redmond, WA) spreadsheets.

Mitochondrial membrane potential. Changes in mitochondrial potential in individual neurons were monitored using the cationic fluorescent dye rhodamine-123 (Molecular Probes), as described previously (Ward et al., 2000). Cells plated on 12 -mm-diameter coverslips were incubated at $37^{\circ} \mathrm{C}$ for 25 min with $2.6 \mu \mathrm{M}$ rhodamine-123 to achieve a quenching concentration of dye within the mitochondrial matrix. Coverslips were washed and placed in a BSS-containing open-bath imaging chamber (Warner Instruments, Hamden, CT) maintained at $37^{\circ} \mathrm{C}$. Single-cell imaging was performed using an Olympus IX70 inverted epifluorescence microscope with a $40 \times$ oil immersion objective (Olympus America, Melville, NY). The dye was excited at $485 \mathrm{~nm}$ using a Spectramaster monochromator (PerkinElmer, Cambridge, UK), and fluorescence was measured using a fura-2/rhodamine dichroic mirror and emission filter set (Chroma Technology, Brattleboro, VT). Images were collected and analyzed using the Imaging Suite software (Olympus America). Data were recorded for 5-10 individual neurons per coverslip and from multiple coverslips. The extent of the barbiturate-induced mitochondrial membrane depolarization was estimated using a mathematical simulation of single-cell fluorescent responses, as described previously (Ward et al., 2000). The following parameters were assumed for the simulation: resting plasma membrane potential of $-60 \mathrm{mV}$; initial mitochondrial membrane potential $\left(\Delta \psi_{\mathrm{m}}\right)$ of $-150 \mathrm{mV}$; cell volume occupied by matrix, $1 \%$; permeability constant for plasma membrane equilibration, $0.0003 \mathrm{sec}^{-1}$; quench limit for probe in the matrix, $20 \mu \mathrm{M}$; and estimated extracellular probe concentration, $80 \mathrm{~nm}$.

Whole-cell patch-clamp recording. Current recordings were obtained using the whole-cell patch-clamp technique at room temperature. The pipette solution contained (in $\mathrm{mM}$ ): $135 \mathrm{KCl}, 3 \mathrm{Mg}$-ATP, 5 EGTA, and 10 HEPES, pH 7.2, with KOH. The external solution was $\mathrm{Mg}^{2+}$-free BSS containing $1 \mu \mathrm{M}$ glycine to potentiate NMDA-induced currents. For both pipette and external solutions, osmolarity was adjusted to $300 \mathrm{mOsm}$ with sucrose. Pipettes had resistance between 4 and $6 \mathrm{M} \Omega$. Series resistance was $<10 \mathrm{~m} \Omega$ and was compensated to the maximum extent possible (usually $>60 \%$ ) without causing oscillation or overshoot. Connection of the perfusion chamber to ground was established via a $3 \mathrm{M} \mathrm{KCl}$ agarose bridge. The U-tube method (Duan and Cooke, 1999) was used for rapid solution change.

Statistics. Statistical differences between experimental groups were determined by performing one-way ANOVA followed, where applicable, by Dunnett's test for multiple comparisons against a control group or Student Newman-Keuls test for comparisons between multiple experimental groups.

\section{RESULTS}

\section{Barbiturates potentiate NMDA neurotoxicity through actions at an intracellular site}

Previous studies have shown that secobarbital at high concentrations (1-3 $\mathrm{mm}$ ) can reduce NMDA-mediated neuronal death (Giffard et al., 1993). To more fully define the effects of barbiturates on NMDA toxicity, we measured NMDA-induced neuronal death over a range of NMDA and secobarbital concentrations. Using NMDA concentrations that produced a moderate degree of neuron death $(<50 \%)$ (Fig. $1 A)$, we found that secobarbital concentrations in the 100-300 $\mu \mathrm{M}$ range amplified NMDA toxicity, with this effect becoming less pronounced at higher secobarbital concentrations (Fig. 1B,C). The more lipid-soluble thiobarbiturate, thiamylal, also displayed a biphasic effect on NMDA toxicity, with NMDA-induced neuron death potentiated by $30 \mu \mathrm{M}$ thiamylal but significantly reduced by $1 \mathrm{~mm}$ thiamylal (Fig. $1 C$ ). Propidium iodide staining performed in cultures $24 \mathrm{hr}$ after NMDA and barbiturate exposures confirmed that only neuronal death contributed to the $\mathrm{LDH}$ signal (data not shown). Figure $1 C$ also shows that the increase in NMDA toxicity produced by secobarbital and thiamylal was eliminated by the addition of 10 $\mu \mathrm{M}$ (+)-5-methyl-10,11-dihydro-5H-dibenzo [a,d] cyclohepten5,10-imine maleate (MK-801), a noncompetitive NMDA receptor antagonist. Together with the lack of toxicity of the barbiturates alone, these results suggest that the barbiturates may act by 

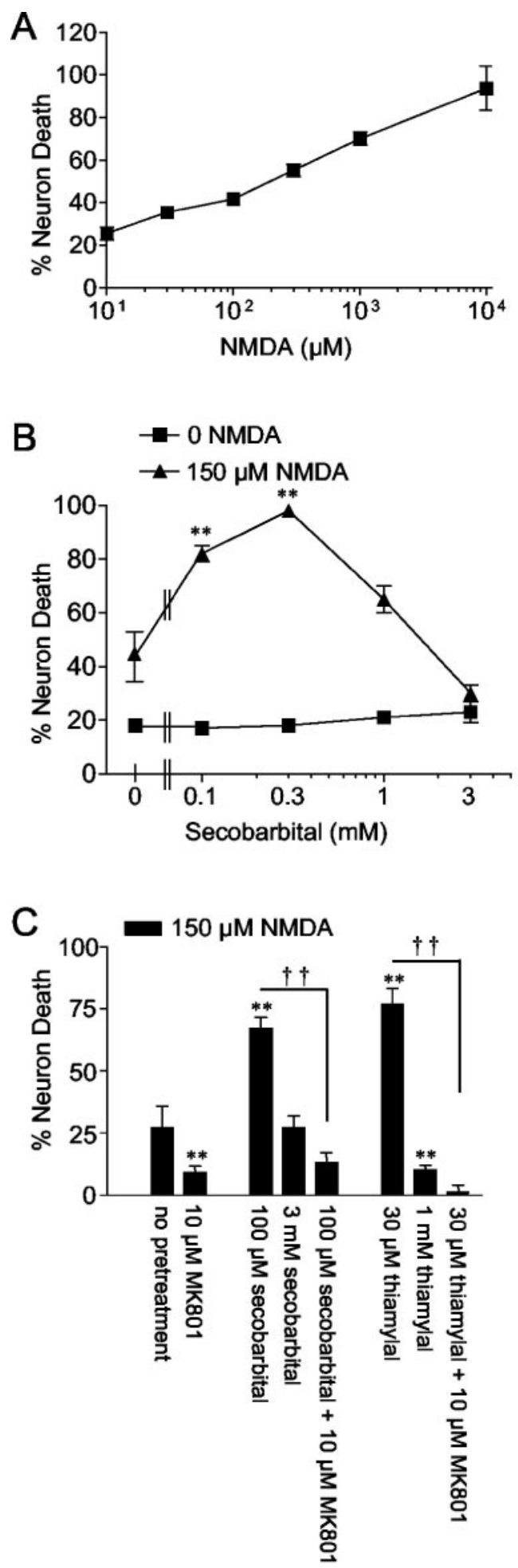

Figure 1. Effects of barbiturates on NMDA neurotoxicity. $A$, Exposure to NMDA for 5 min resulted in neuronal death with an $\mathrm{EC}_{50}$ of $\sim 200 \mu \mathrm{M}$. $B$, Secobarbital at 100 and $300 \mu \mathrm{M}$ potentiated NMDA neurotoxicity, but this effect was reversed at higher secobarbital concentrations. Vertical hatches mark the beginning of the $\log$ scale. $* * p<0.01$ versus the 0 secobarbital control in each of the two treatment groups; $n \geq 7$. $C$, The thiobarbiturate thiamylal produced a similar biphasic effect. For both thiamylal and secobarbital, MK-801 completely blocked the toxicity produced by NMDA plus barbiturate. $* * p<0.01$ versus the 0 barbiturate group; ${ }^{\dagger} p<0.01$ between indicated groups; $n \geq 7$. All data are means \pm SE. $C$, Data are shown after subtraction of $12 \%$ neuronal death observed in the control (wash only) condition. potentiating NMDA toxicity specifically. This was supported by an additional set of studies that showed that secobarbital had a negligible effect on $\mathrm{H}_{2} \mathrm{O}_{2}$-induced neuronal death. The $\mathrm{LD}_{50}$ for $\mathrm{H}_{2} \mathrm{O}_{2}$ was $\sim 50 \mu \mathrm{M}$ in both the presence and absence of $100 \mu \mathrm{M}$ secobarbital (data not shown).

To determine whether barbiturates amplify NMDA neurotoxicity through actions at the plasma membrane or at intracellular sites, experiments were also performed using $\mathrm{N}$-glucoside amobarbital, a hydrophilic $N$-glucoside derivative of amobarbital with limited plasma membrane permeability (Tang, 1990). $N$-glucoside amobarbital did not potentiate NMDA toxicity but instead exhibited only neuroprotective effects (Fig. $2 A$ ). In contrast, the parent compound amobarbital had a biphasic effect on NMDA-induced cell death over the same concentration range (Fig. $2 A$ ), similar to that observed with secobarbital and thiamylal. The failure of $N$-glucoside amobarbital to amplify NMDA toxicity suggests that barbiturates potentiate NMDA toxicity through actions at an intracellular site. Additionally, the marked reduction in NMDA toxicity observed with $N$-glucoside amobarbital suggests that the reduction in NMDA toxicity observed with high concentrations $(>300 \mu \mathrm{M})$ of the standard, cell-permeant barbiturates may be mediated by actions at the neuronal cell membrane. These possibilities were also investigated by measuring the effects of barbiturates on NMDA-induced currents in whole-cell patch-clamped neurons. Secobarbital reduced peak NMDA-induced current in a monophasic, concentration-dependent manner, achieving a $61 \pm$ $9 \%$ reduction at $1 \mathrm{~mm}$ (Fig. $2 B, C$ ). $N$-glucoside amobarbital produced a similar inhibition of NMDA-induced currents (Fig. $2 B, C)$.

\section{Barbiturates depolarize mitochondria by inhibiting mitochondrial ATP synthesis}

Previous studies have suggested that mitochondria are an intracellular site of barbiturate action (Aldrich and Parker, 1960). Barbiturate effects on mitochondrial membrane potential $\left(\Delta \psi_{\mathrm{m}}\right)$ were assessed in individual neurons using the fluorescent cationic dye rhodamine-123. In 25 of 29 neurons analyzed, secobarbital $(100 \mu \mathrm{M})$ caused significant mitochondrial depolarization (Fig. $3 A$ ), as reflected by an increase in total cell fluorescence caused by release of rhodamine-123 from the quenched environment of the matrix (for review, see Nicholls and Ward, 2000). The dye quench limit was surpassed because carbonyl cyanide $p$-trifluoromethoxyphenylhydrazone (FCCP) was able to increase whole-cell fluorescence (data not shown). Subsequent application of the ATP synthase inhibitor oligomycin $(2.9 \mu \mathrm{g} / \mathrm{ml})$ produced additional mitochondrial depolarization (Fig. $3 A$ ), indicating that $\Delta \psi_{\mathrm{m}}$ had been sustained by "reverse" operation of ATP synthase. Oligomycin added to cells with normal $\Delta \psi_{\mathrm{m}}$ produced either no change in $\Delta \psi_{\mathrm{m}}$ or a slight hyperpolarization consistent with slowed proton influx through the mitochondrial ATP synthase complex (Fig. 3B). As expected, the cell-impermeant barbiturate $N$-glucoside amobarbital had no effect on $\Delta \psi_{\mathrm{m}}$ (Fig. $3 B$ ), whereas the parent compound amobarbital produced significant depolarization (data not shown).

Mitochondrial depolarization with reduced ATP synthesis can result from impaired electron transport (or substrate supply) or enhanced inner membrane proton permeability. In cells with active glycolysis, the former will result in only a partial depolarization, because ATP synthase reversal can maintain a suboptimal membrane potential even in the face of total respiratory inhibition by consuming ATP generated by glycolysis. In contrast, increasing concentrations of a protonophore would decrease $\Delta \psi_{\mathrm{m}}$ 


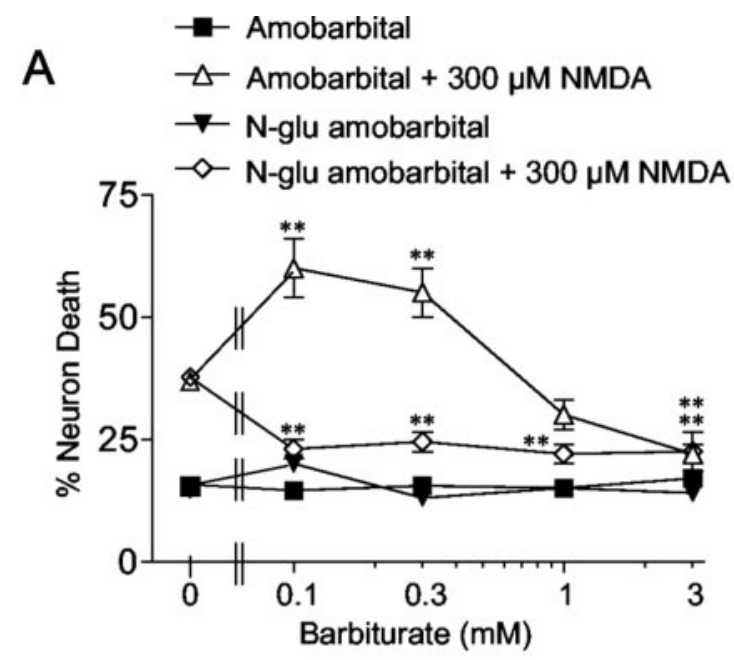

Secobarbital

B
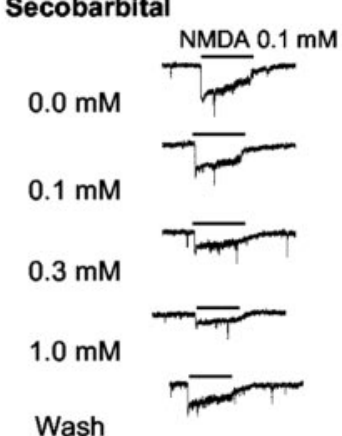

Wash

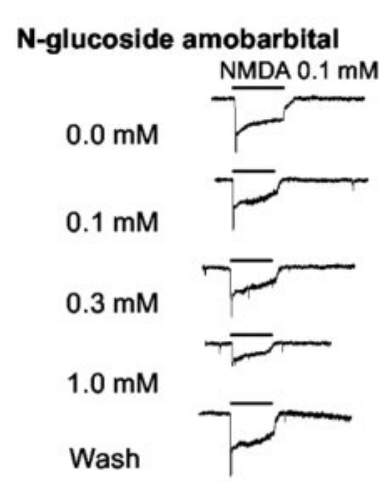

100 pA $\frac{}{20 \mathrm{msec}}$

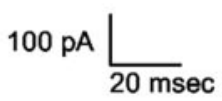

C

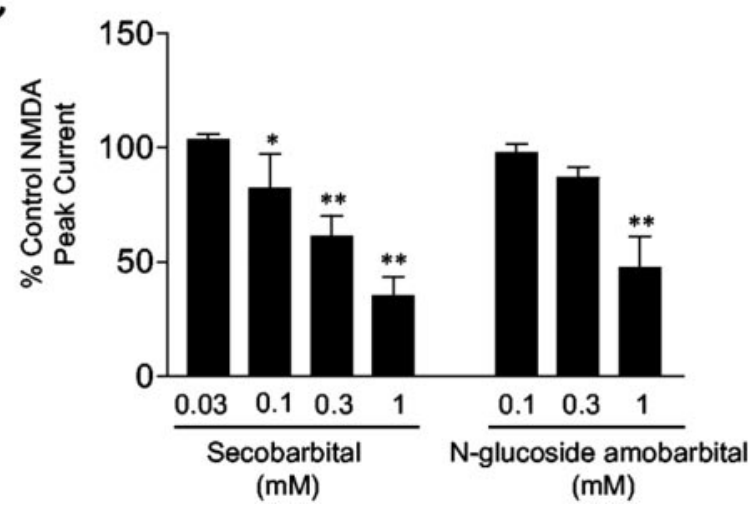

Figure 2. Comparisons between a cell-permeant and a cell-impermeant barbiturate. $A$, The cell-impermeant barbiturate $N$-glucoside $(N$-glu) amobarbital reduced NMDA toxicity at all concentrations tested, whereas the parent compound had a biphasic effect on NMDA toxicity. Vertical hatches mark the beginning of the log scale. $B$, Representative patch-clamp recordings from different neurons exposed to NMDA in the presence of sequentially elevated concentrations of secobarbital or $N$-glucoside amobarbital. $C$, Pooled data showing a concentration-dependent inhibition of the NMDA current with both barbiturates. Currents from each recorded neuron are normalized to the peak current recorded in the absence of barbiturate. Data are means $\pm \mathrm{SE} ; * p<0.05 ; * * p<0.01$ versus control; $n=3-6$.

asymptotically toward zero. In an attempt to distinguish between these alternatives, the relationship between mitochondrial depolarization and secobarbital concentration was compared with that

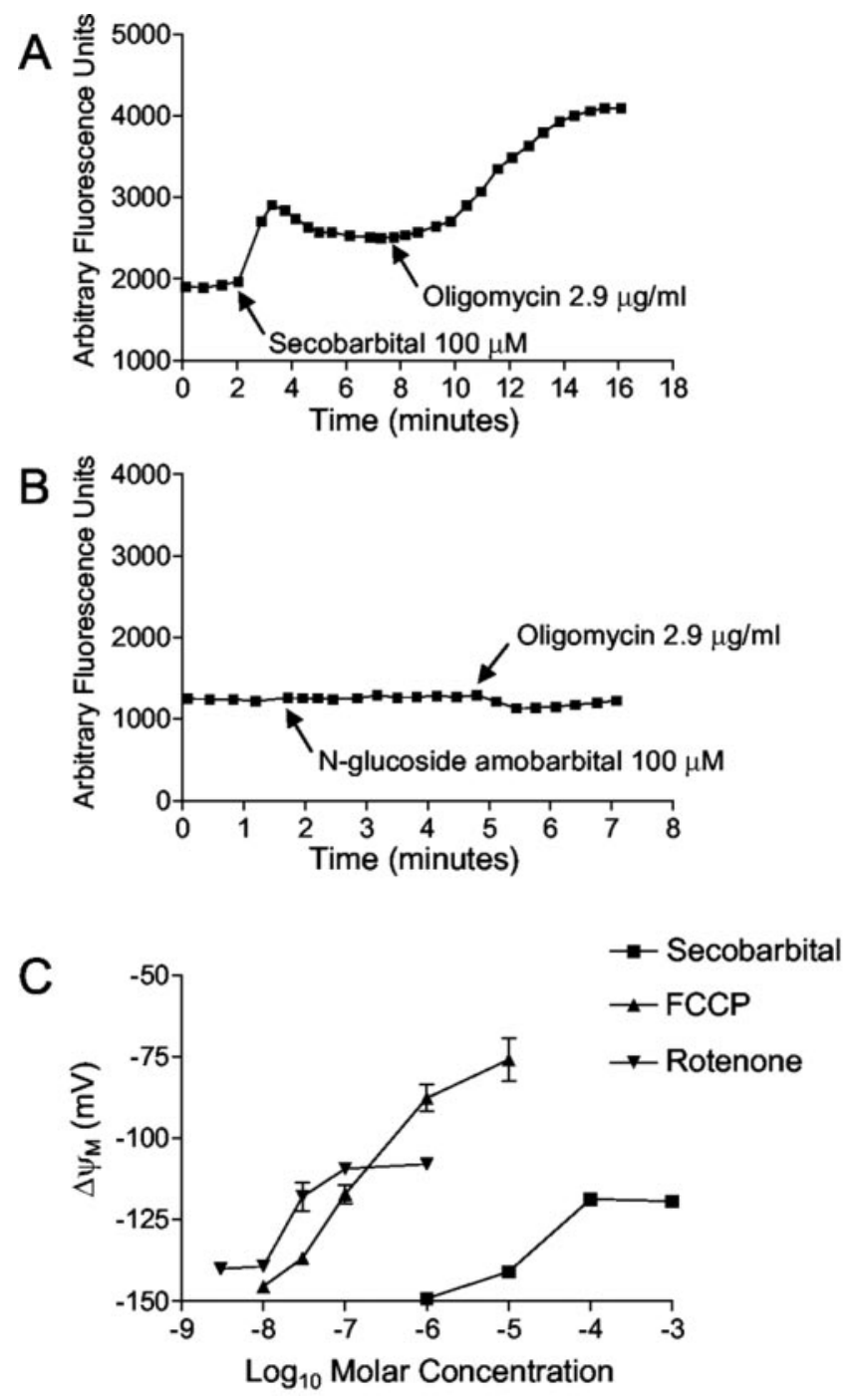

Figure 3. Barbiturates cause mitochondrial depolarization in situ. A, Secobarbital induced an increase in total cell rhodamine-123 fluorescence, indicative of mitochondrial depolarization. The addition of oligomycin caused additional depolarization, indicating that secobarbital induces ATP synthase reversal. $B$, The cell-impermeable barbiturate $N$-glucoside amobarbital had no effect on $\Delta \Psi_{\mathrm{m}}$. Oligomycin caused a slight membrane hyperpolarization. $C$, Secobarbital and the complex 1 inhibitor rotenone increased peak mitochondrial depolarization to a plateau at approximately $-110 \mathrm{mV}$, whereas the uncoupler FCCP increased peak depolarization dose dependently. The $\mathrm{EC}_{50}$ for secobarbital is $\sim 30 \mu \mathrm{M}$. Data in $A$ and $B$ are representative traces. Data in $C$ are means $\pm \mathrm{SE}$ of $\geq 10$ neurons from two different coverslips.

of a known mitochondrial complex 1 inhibitor (rotenone) and an established uncoupler, FCCP. Figure $3 C$ shows that both rotenone and secobarbital produced a concentration-dependent mitochondrial depolarization. This plateaued at a residual mitochondrial membrane potential of approximately $-110 \mathrm{mV}$, as estimated by a simulation that predicts $\Delta \psi_{\mathrm{m}}$ from whole-cell fluorescence intensity (Ward et al., 2000). In contrast, increasing concentrations of FCCP produced a near linear depolarization with no observable plateau. Because maximal inhibition of respiration produces only partial mitochondrial depolarization, secobarbital displayed characteristics of a respiration inhibitor.

Because barbiturates were found to potentiate NMDA neurotoxicity, we also assessed the effect of barbiturates on NMDA- 

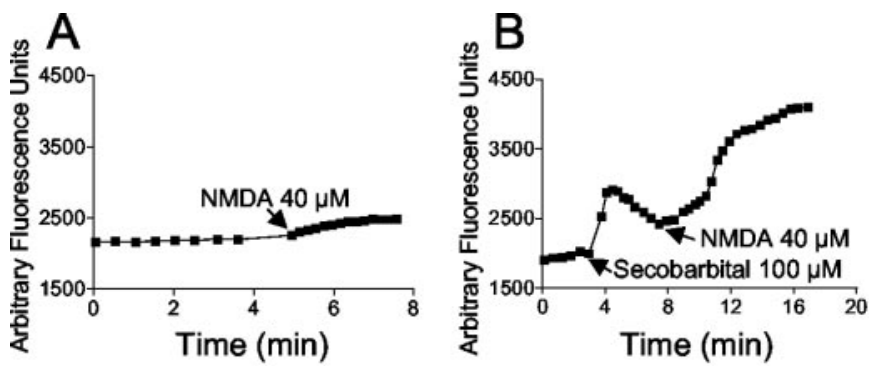

Figure 4. Secobarbital potentiates NMDA-induced mitochondrial depolarization. $A$, Bath-applied NMDA $(40 \mu \mathrm{M})$ produced an increase in total cell rhodamine-123 fluorescence, indicative of a slight mitochondrial depolarization. $B$, Pretreatment with $100 \mu \mathrm{M}$ secobarbital enhanced NMDA-induced depolarization. Data are representative traces of 12 individual neurons from two separate coverslips for each treatment regimen.

induced changes in $\Delta \psi_{\mathrm{m}}$. NMDA alone caused a small mitochondrial depolarization (Fig. 4A), indicative of increased mitochondrial $\mathrm{Ca}^{2+}$ uptake (Ward et al., 2000). In the presence of $100 \mu \mathrm{M}$ secobarbital, the NMDA-induced mitochondrial depolarization was greatly increased (Fig. 4B). This result is congruent with the increased neurotoxicity of NMDA in the presence of $100 \mu \mathrm{M}$ secobarbital (Fig. 1).

\section{Barbiturates enhance NMDA-induced intracellular calcium elevations}

Mitochondrial dysfunction can lead to neuron death by disrupting $\mathrm{Ca}^{2+}$ homeostasis (Stout et al., 1998; Murphy et al., 1999; Nicholls and Ward, 2000; Sattler and Tymianski, 2000). NMDAinduced $\mathrm{Ca}^{2+}$ elevations were monitored in the presence or absence of secobarbital using ratiometric fura- 2 imaging. Barbiturates had biphasic, concentration-dependent effects on free $\mathrm{Ca}^{2+}$ that paralleled their effects on NMDA-induced cell death. As shown in Figure $5 A$, NMDA applied at a concentration that normally induced a negligible increase in intracellular $\mathrm{Ca}^{2+}(5$ $\mu \mathrm{M}$ ) was found to induce a large $\mathrm{Ca}^{2+}$ increase when applied in the presence of $100 \mu \mathrm{M}$ secobarbital but not $1 \mathrm{~mm}$ secobarbital. When NMDA was applied at a concentration sufficient to induce a substantial $\mathrm{Ca}^{2+}$ elevation $(20 \mu \mathrm{M})$, this increase was attenuated in the presence of $1 \mathrm{~mm}$ amobarbital, $1 \mathrm{~mm} N$-glucoside amobarbital (Fig. 5B), or $1 \mathrm{~mm}$ secobarbital (data not shown).

\section{DISCUSSION}

These findings suggest that barbiturates potentiate NMDA neurotoxicity by inhibiting mitochondrial respiration and thereby amplifying NMDA-induced mitochondrial depolarization and intracellular $\mathrm{Ca}^{2+}$ dysregulation. NMDA neurotoxicity was increased by all three barbiturates tested: secobarbital, amobarbital, and thiobarbital. Thiamylal was the most potent of these, but all three agents showed maximal effects in the 30-100 $\mu \mathrm{M}$ concentration range. Because these agents represent members of both the oxybarbiturate and thiobarbiturate classes, the findings suggest that potentiation of NMDA toxicity is likely a general property common to all cell-permeant barbiturates.

Importantly, the poorly permeant congener $N$-glucoside amobarbital did not potentiate NMDA toxicity, and this provides one line of evidence that barbiturate influences on NMDA toxicity occur at an intracellular site. $N$-glucoside amobarbital was originally synthesized for studies of barbiturate metabolism (Soine et al., 1986, 1991). The pharmacology of this compound has not been extensively characterized, and it remains possible that its
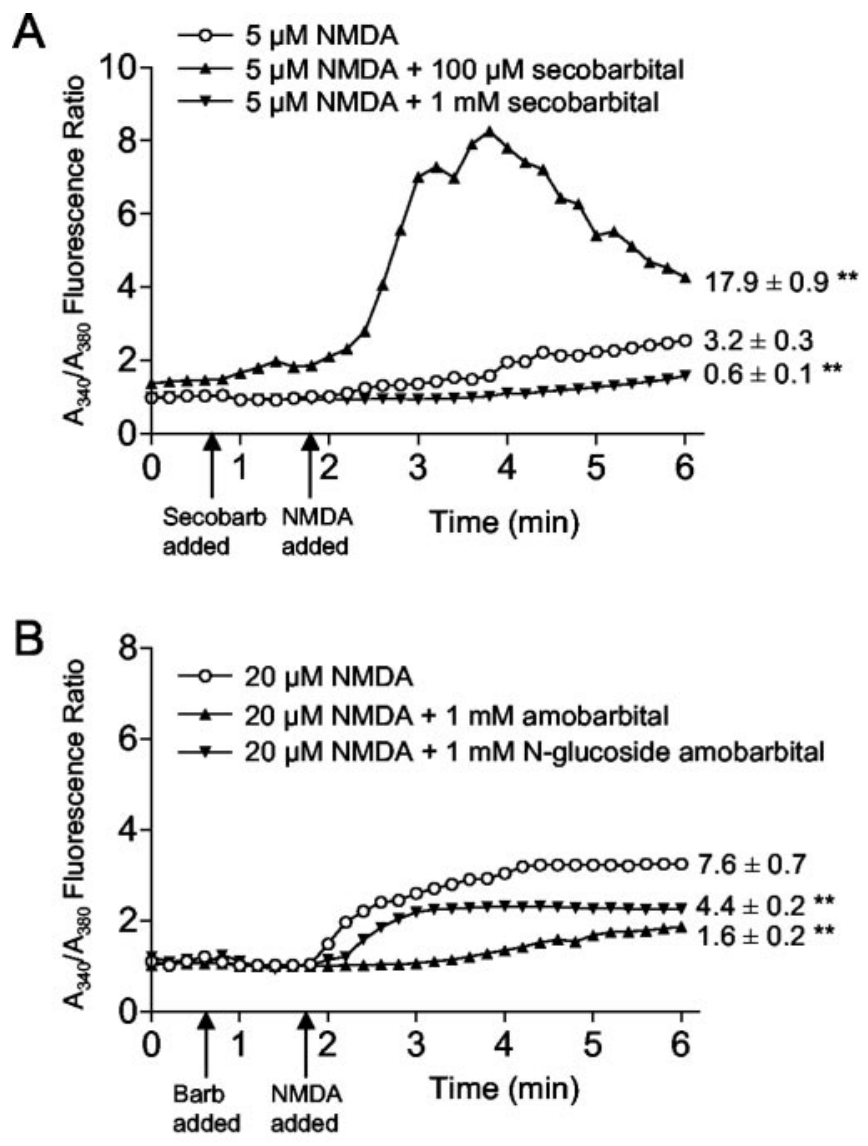

Figure 5. Barbiturate effects on NMDA-induced cytoplasmic $\mathrm{Ca}^{2+}$ elevations. $A$, A $5 \mu \mathrm{M}$ concentration of NMDA alone produced a small rise in intracellular $\mathrm{Ca}^{2+}$. This increase was significantly increased in the presence of $100 \mu \mathrm{M}$ secobarbital but nearly eliminated in the presence of $1 \mathrm{~mm}$ secobarbital. Secobarb, Secobarbital. B, The larger increase in intracellular $\mathrm{Ca}^{2+}$ induced by $20 \mu \mathrm{M}$ NMDA was reduced by both $1 \mathrm{~mm}$ amobarbital and $1 \mathrm{~mm} N$-glucoside amobarbital. Error bars are omitted for clarity. Values adjacent to the tracings are the mean \pm SE of the integrated NMDA-induced changes in the fura- 2 fluorescence ratio integrated over the 4 min interval beginning $10 \mathrm{sec}$ after the addition of NMDA. $* * p<0.01$ versus NMDA alone; $n=35-49$ in $A ; n=12-19$ in B. Barb, Barbiturate.

failure to amplify the effects of NMDA is attributable to some property other than poor cell entry. However, the studies presented here show that $N$-glucoside amobarbital is not inert. $N$-glucoside amobarbital was found to attenuate NMDA toxicity over the same concentration range that other barbiturates, including amobarbital, had the opposite effect. Moreover, $N$-glucoside amobarbital also attenuated NMDA-induced intracellular $\mathrm{Ca}^{2+}$ elevations and blocked NMDA-induced currents in whole-cell patch-clamped neurons. These results suggest that the neuroprotective effects of $\mathrm{N}$-glucoside amobarbital occur at an extracellular site, whereas the NMDA-potentiating effects of permeable barbiturates are intracellular.

Previous studies have shown that barbiturates exacerbate neuronal cell death induced by glucose deprivation but not by oxygen deprivation (Giffard et al., 1993). These studies also showed that 100-300 $\mu \mathrm{M}$ secobarbital amplified ATP depletion during glucose deprivation but not oxygen deprivation. One explanation for these results is that barbiturate inhibition of mitochondrial respiration (Aldrich and Parker, 1960) is sufficient to limit oxidative ATP production. Barbiturate effects on mitochondria could like- 
wise mediate exacerbation of NMDA neurotoxicity, in accord with effects of other known mitochondrial inhibitors (White and Reynolds, 1995; Greene et al., 1998). In support of this idea, results obtained in this study provide the first direct evidence that barbiturates produce mitochondrial membrane depolarization in neurons. For secobarbital, a maximal depolarization was achieved at $100 \mu \mathrm{M}$, and the $\mathrm{EC}_{50}$ was $\sim 30 \mu \mathrm{M}$, which corresponds to the concentrations at which secobarbital potentiated NMDA neurotoxicity.

Mitochondrial depolarization can result from increased ATP demand or increased $\mathrm{Ca}^{2+}$ influx (Nicholls and Ward, 2000). In both cases, mitochondrial ATP synthase continues to function and support the cytosolic ATP:ADP ratio. Alternatively, mitochondrial depolarization can result from enhanced inner membrane proton permeability (classical uncoupling) or from impaired respiration. In these conditions, cytosolic ATP is consumed by ATP synthase as it functions in reverse mode to extrude matrix protons and maintain $\Delta \psi_{\mathrm{m}}$. In the present studies, the ATP synthase inhibitor oligomycin increased mitochondrial depolarization in neurons treated with secobarbital, indicating that ATP synthase was functioning in reverse mode and that $\Delta \psi_{\mathrm{m}}$ was being supported by cytoplasmic ATP.

The mechanism for barbiturate-induced disruption of ATP synthesis was probed by comparing secobarbital to a known protonophoric uncoupler (FCCP) and a known respiration inhibitor (rotenone). High protonophore concentrations can totally collapse $\Delta \psi_{\mathrm{m}}$. In contrast, respiratory chain inhibitors, even after $100 \%$ blockade of electron transport, only partially depolarize $\Delta \psi_{\mathrm{m}}$ caused by ATP synthase reversal. Thus, the plot of mitochondrial depolarization versus concentration for rotenone reaches a submaximal plateau, as depicted in Figure $3 C$. An analogous plot for secobarbital revealed a maximal depolarizing capability similar to rotenone, between 30 and $100 \mu \mathrm{M}$. This suggests that secobarbital is an inhibitor of mitochondrial respiration, in agreement with classical studies using isolated mitochondria (Aldrich and Parker, 1960). The single-cell fluorescence simulation (Ward et al., 2000) suggests a maximal secobarbitalinduced depolarization of $\sim 25 \mathrm{mV}$.

The effects of secobarbital on NMDA-induced mitochondrial depolarization were paralleled by its effects on intracellular free $\mathrm{Ca}^{2+}$ concentrations. Secobarbital alone caused little or no elevation in intracellular $\mathrm{Ca}^{2+}$, but when present during NMDA exposure, it increased the cytoplasmic $\mathrm{Ca}^{2+}$ concentrations by several-fold. This result is consistent with previous studies showing that disrupted intracellular $\mathrm{Ca}^{2+}$ homeostasis correlates with the extent of glutamate-induced mitochondrial depolarization (Vergun et al., 1999; Ward et al., 2000). The NMDA concentrations used for the studies of intracellular free $\mathrm{Ca}^{2+}$ changes were lower than those used for the cell death studies because of the concern that higher NMDA concentrations would saturate the high-affinity fura-2 calcium indicator (Hyrc et al., 1997). It is possible that $\mathrm{Ca}^{2+}$ elevations produced by higher NMDA concentrations might not exhibit the same degree of amplification in the presence of barbiturates, but this possibility seems unlikely given that the barbiturate influences on NMDA-induced cell death, mitochondrial membrane depolarization, and calcium elevations were all consistent.

Barbiturates have numerous effects on cell metabolism, and consequently, there are several ways, both direct and indirect, that barbiturates could influence mitochondrial function and NMDA neurotoxicity. The results provided here and in previous studies indicate that barbiturates act by a direct effect on mito- chondrial electron transport. It has been known since the 1960s that barbiturates can block complex 1 activity in isolated liver mitochondria at submillimolar concentrations (Aldrich and Parker, 1960; Chance and Hollunger, 1963). The present studies show that secobarbital has the same pattern of effects on the mitochondrial membrane potential of intact neurons as the complex 1 inhibitor rotenone (although rotenone is more potent). A causative link between the effects of barbiturates on neuronal mitochondria and their potentiating effect on NMDA toxicity is supported by the fact that these effects, as well as the amplification of NMDA-induced calcium elevations, are all observed over the 30-100 $\mu \mathrm{M}$ range. Together, these observations support the idea that barbiturates act in a manner similar to other mitochondrial inhibitors (Novelli et al., 1988; Beal, 1992; Greene and Greenamyre, 1996; Greene et al., 1998) to potentiate NMDAinduced calcium elevations and neurotoxicity. It remains possible, however, that there are additional effects of barbiturates that also contribute to these processes or that the increased calcium elevations are not directly related to increased neuronal death.

Our findings also identify a second mechanism by which barbiturates influence excitotoxicity. At concentrations of $\geq 1 \mathrm{~mm}$, all of the barbiturates tested were found to attenuate NMDAinduced inward currents. These observations are consistent with previous reports (Daniell, 1994; Charlesworth et al., 1995) and may reconcile the opposing effects of micromolar and millimolar barbiturate concentrations on excitotoxicity; barbiturates in the 30-100 $\mu \mathrm{M}$ range exacerbate excitotoxicity by impairing mitochondrial respiration, but this effect becomes moot at millimolar barbiturate concentrations, because flux through the NMDA receptor complex is then effectively blocked. An additional contributory factor may be that the effect of barbiturates on mitochondrial membrane potential plateaus at $\sim 100 \mu \mathrm{M}$ and does not increase further at the higher barbiturate concentrations (Fig. 3).

Although effects of barbiturates on mitochondrial energy metabolism are well established, they have not been recognized previously as physiologically significant in neurons. Studies with cardiac muscle provide a precedent for functionally significant effects resulting from barbiturate-induced mitochondrial inhibition. Bhayana et al. (1980) showed in perfused rat hearts that pentobarbital and thiopental reduce both state 3 respiration and myocardial contractile force at $0.1-0.5 \mathrm{~mm}$. Although other mechanisms could not be excluded, it is likely that the impaired respiration contributed to the impaired contractility, because cardiac muscle relies almost exclusively on oxidative ATP production. Similarly, ATP levels and glutamate uptake were shown to be reduced in astrocytes exposed to barbiturates at submillimolar concentrations (Swanson et al., 1997). These effects were strikingly potentiated by glucose deprivation, consistent with a greater reliance of astrocytes on glycolytic rather than oxidative metabolism (Swanson, 1992; Silver et al., 1997). Studies of brain and kidney in situ have demonstrated elevated cellular $\beta$-nicotinamide adenine dinucleotide, reduced form (NADH) levels during barbiturate inf usions, consistent with respiratory chain inhibition, but did not examine the effects of this impairment on cell function (Chance et al., 1962). The present study provides the first demonstration that barbiturate inhibition of neuronal mitochondrial function can become functionally significant during NMDA receptor stimulation.

Barbiturates are widely used in studies of neuronal function. The present findings suggest that interpretation of experiments using barbiturates should include consideration of mitochondrial function and cellular energetics in addition to effects on excitable 
membranes. Barbiturates are also widely used as neuroprotective agents (Cheng et al., 1997). The barbiturate dose range shown to potentiate NMDA-mediated neuronal death in this study is within the range of barbiturate concentrations achieved in clinical use. Barbiturate treatment of refractory status epilepticus targets EEG burst suppression as a treatment goal (Lowenstein et al., 1988; Van Ness, 1990). Plasma thiopental concentrations of 80$200 \mu \mathrm{M}$ produce a burst suppression EEG pattern in human subjects (Airey et al., 1982; Stanski et al., 1984), which is considered by many authors to signal the optimal anesthesia depth for neuroprotection (Zaidan et al., 1991; Frawley et al., 1994). Barbiturate concentrations in CSF are typically $15-40 \%$ of serum levels, and a clinical study found that the CSF concentration of thiopental required to produce an anticonvulsant effect in a patient with status epilepticus was 15-90 $\mu \mathrm{M}$ (Airey et al., 1982). In addition, brain concentrations of barbiturates increase with extended use because of tolerance, requiring dose escalation, and because of accumulation in lipids (Bolander et al., 1984; Koskela and Wahlström, 1989). Whether barbiturate potentiation of excitotoxicity is sufficient to produce a net negative effect on neuronal survival in vivo is not clear; however, the present studies suggest that barbiturates could exacerbate excitotoxic injury, particularly if glucose supply is impaired.

\section{REFERENCES}

Airey IL, Smith PA, Stoddart JC (1982) Plasma and cerebrospinal fluid barbiturate levels during prolonged continuous thiopentone infusion. Anaesthesia 37:328-331.

Aldrich WN, Parker VH (1960) Barbiturates and oxidative phosphorylation. Biochem J 76:47-56.

Barker JL, Ransom BR (1978) Pentobarbitone pharmacology of mammalian central neurones grown in tissue culture. J Physiol (Lond) 280:355-372.

Beal MF (1992) Does impairment of energy metabolism result in excitotoxic neuronal death in neurodegenerative illnesses? Ann Neurol 31:119-130.

Bhayana V, Alto LE, Dhalla NS (1980) Effects of pentobarbital and pentothal on rat heart contractile force and oxidative phosphorylation activities. Gen Pharmacol 11:375-377.

Bolander HG, Wahlström G, Norberg L (1984) Reevaluation of potency and pharmacokinetic properties of some lipid-soluble barbiturates with an EEG-threshold method. Acta Pharmacol Toxicol (Copenh) 54:33-40.

Chance B, Hollunger G (1963) Inhibition of electron and energy transfer in mitochondria. J Biol Chem 278:418-431.

Chance B, Cohen P, Jobsis F, Schoener B (1962) Intracellular oxidationreduction states in vivo. Science 137:499-508.

Charlesworth P, Jacobson I, Richards CD (1995) Pentobarbitone modulation of NMDA receptors in neurones isolated from the rat olfactory brain. Br J Pharmacol 116:3005-3013.

Cheng MA, Theard MA, Tempelhoff R (1997) Intravenous agents and intraoperative neuroprotection. Beyond barbiturates. Crit Care Clin 13:185-199.

Daniell LC (1994) Effect of anesthetic and convulsant barbiturates on $N$-methyl-D-aspartate receptor-mediated calcium flux in brain membrane vesicles. Pharmacology 49:296-307.

Duan S, Cooke IM (1999) Selective inhibition of transient K+ current by $\mathrm{La} 3+$ in crab peptide-secretory neurons. J Neurophysiol 81:1848-1855.

Edidin M (1970) A rapid, quantitative fluorescence assay for cell damage by cytotoxic antibodies. J Immunol 104:1303-1306.

Frawley JE, Hicks RG, Horton DA, Gray LJ, Niesche JW, Matheson JM (1994) Thiopental sodium cerebral protection during carotid endarterectomy: perioperative disease and death. J Vasc Surg 19:732-738.

Giffard RG, Weiss JH, Swanson RA, Choi DW (1993) Secobarbital attenuates excitotoxicity but potentiates oxygen-glucose deprivation neuronal injury in cortical cell culture. J Cereb Blood Flow Metab 13:803-810.

Greene JG, Greenamyre JT (1996) Bioenergetics and glutamate excitotoxicity. Prog Neurobiol 48:613-634.

Greene JG, Sheu SS, Gross RA, Greenamyre JT (1998) 3-Nitropro- pionic acid exacerbates $N$-methyl-D-aspartate toxicity in striatal culture by multiple mechanisms. Neuroscience 84:503-510.

Hyrc K, Handran SD, Rothman SM, Goldberg MP (1997) Ionized intracellular calcium concentration predicts excitotoxic neuronal death: observations with low-affinity fluorescent calcium indicators. J Neurosci 17:6669-6677.

Koh JY, Choi DW (1987) Quantitative determination of glutamate mediated cortical neuronal injury in cell culture by lactate dehydrogenase efflux assay. J Neurosci Methods 20:83-90.

Koskela T, Wahlström G (1989) Comparison of anaesthetic and kinetic properties of thiobutabarbital, butabarbital and hexobarbital after intravenous threshold doses in the male rat. Pharmacol Toxicol 64:308-313.

Lowenstein DH, Aminoff MJ, Simon RP (1988) Barbiturate anesthesia in the treatment of status epilepticus: clinical experience with 14 patients. Neurology 38:395-400.

Murphy AN, Fiskum G, Beal MF (1999) Mitochondria in neurodegeneration: bioenergetic function in cell life and death. J Cereb Blood Flow Metab 19:231-245.

Nicholls DG, Ward MW (2000) Mitochondrial membrane potential and neuronal glutamate excitotoxicity: mortality and millivolts. Trends Neurosci 23:166-174.

Novelli A, Reilly JA, Lysko PG, Henneberry RC (1988) Glutamate becomes neurotoxic via the $N$-methyl-D-aspartate receptor when intracellular energy levels are reduced. Brain Res 451:205-212.

Sattler R, Tymianski M (2000) Molecular mechanisms of calciumdependent excitotoxicity. J Mol Med 78:3-13.

Silver IA, Deas J, Erecinska M (1997) Ion homeostasis in brain cells: differences in intracellular ion responses to energy limitation between cultured neurons and glial cells. Neuroscience 78:589-601.

Soine WH, Soine PJ, Overton BW, Garrettson LK (1986) Product enantioselectivity in the $N$-glucosylation of amobarbital. Drug Metab Dispos 14:619-621.

Soine WH, Soine PJ, England TM (1991) Barbital $N$-glucoside is not detected as a urinary excretion product of barbital in humans. J Pharm Biomed Anal 9:747-752.

Sokoloff L, Reivich M, Kennedy C, Des Rosiers MH, Patlak CS, Pettigrew KD, Sakurada O, Shinohara M (1977) The $\left[{ }^{14} \mathrm{C}\right] d e o x y g l u c o s e$ method for the measurement of local cerebral glucose utilization: theory, procedure, and normal values in the conscious and anesthetized albino rat. J Neurochem 28:897-916.

Stanski DR, Hudson RJ, Homer TD, Saidman LJ, Meathe E (1984) Pharmacodynamic modeling of thiopental anesthesia. J Pharmacokinet Biopharm 12:223-240.

Steen PA, Michenfelder JD (1980) Mechanisms of barbiturate protection. Anesthesiology 53:183-185.

Stout AK, Raphael HM, Kanterewicz BI, Klann E, Reynolds IJ (1998) Glutamate-induced neuron death requires mitochondrial calcium uptake. Nat Neurosci 1:366-373.

Swanson RA (1992) Astrocyte glutamate uptake during chemical hypoxia in vitro. Neurosci Lett 147:143-146.

Swanson RA, Seid LL (1998) Barbiturates impair astrocyte glutamate uptake. Glia 24:365-371.

Swanson RA, Farrell K, Stein BA (1997) Astrocyte energetics, function, and death under conditions of incomplete ischemia: a mechanism of glial death in the penumbra. Glia 21:142-153.

Tang BK (1990) Drug glucosidation. Pharmacol Ther 46:53-56.

Van Ness PC (1990) Pentobarbital and EEG burst suppression in treatment of status epilepticus refractory to benzodiazepines and phenytoin. Epilepsia 31:61-67.

Vergun O, Keelan J, Khodorov BI, Duchen MR (1999) Glutamateinduced mitochondrial depolarisation and perturbation of calcium homeostasis in cultured rat hippocampal neurones. J Physiol (Lond) 519:451-466.

Ward MW, Rego AC, Frenguelli BG, Nicholls DG (2000) Mitochondrial membrane potential and glutamate excitotoxicity in cultured cerebellar granule cells. J Neurosci 20:7208-7219.

Werz MA, Macdonald RL (1985) Barbiturates decrease voltagedependent calcium conductance of mouse neurons in dissociated cell culture. Mol Pharmacol 28:269-277.

White RJ, Reynolds IJ (1995) Mitochondria and $\mathrm{Na}^{+} / \mathrm{Ca}^{2+}$ exchange buffer glutamate-induced calcium loads in cultured cortical neurons. J Neurosci 15:1318-1328.

Ying W, Han SK, Miller JW, Swanson RA (1999) Acidosis potentiates oxidative neuronal death by multiple mechanisms. J Neurochem $73: 1549-1556$

Zaidan JR, Klochany A, Martin WM, Ziegler JS, Harless DM, Andrews RB (1991) Effect of thiopental on neurologic outcome following coronary artery bypass grafting. Anesthesiology 74:406-411. 